\title{
Opportunities, challenges and pathways of nano- medicines: a concise review
}

Volume I Issue 2 - 2014

\section{Introduction}

The number of poorly soluble drugs and drug candidates rapidly increases. As per one estimate nearly $70 \%$ of molecules developed have low solubility problems and consequently face poor oral bioavailability and delivery problems. At the beginning of the 1990s the drugs based on nano- crystals have been developed as more efficient approach to increase drug solubility and dissolution velocity. After successful trials and tests the first drug was marketed in the year 2000 as Rapamune ${ }^{\circledR}$ an immunosuppressive, by pharmaceutical company Wyeth., ${ }^{1,2}$ Later on, other bigwigs have enter the fray to develop new drugs based on nano formulations, for example, Emend ${ }^{\circledR}$ as an anti-emetic by Merck and Tricor ${ }^{\circledR}$ for hypercholesterolemia by Abbott. Reducing the particle size of an API (Active Pharmaceutical Ingredient) is an efficient and reliable technique of improving the bioavailability of relatively insoluble drug that is often limited by poor dissolution rate. Recently, some attempts have been made to develop the nano crystalline API using micro-emulsion technique and compared the physical properties with the bulk materials, for example, n-butyl 4-(3, 4-dimethoxyphenyl) -6-methyl-2-thioxo-1,2,3,4 etrahydropyrimidine-5-carboxylate. ${ }^{3}$ The nano- structures are expected to give the advantages such as increased rate of absorption, reduction in the required dose, reduction in the fed/ fasted variability, improved dose proportionality, smaller dosage forms, rapid formulation development and possible application to all route of administration and any dosage form. ${ }^{2,4}$

Nano particles have become the cynosure in the front line research for their prophylactic, diagnostic and therapeutic applications. The nano- particles properties offer solutions for major challenges in treating cancer, cardiovascular and neurodegenerative diseases and bring new hopes to millions of sufferers globally. As per the recent trend, the most active areas of nano-medicine research and product development are cancer treatment, imaging contrast agent and biomarker detection. ${ }^{5}$ Further elaborating the applications of nanoparticles one can include the use in vaccination, MRI(Magnetic Resonance Imaging) contrast agent, fluorescent biological labels, pathogen detection, protein identification, DNA and gene delivery agents and separation of biological molecules and cells. ${ }^{6}$

Functionalization as well as encapsulation of nano-particles isan important direction of research. Nano particles can be functionalized to specifically target tumor cells allowing the imaging and therapeutic agents to be delivered directly to the affected cells. These multifunctional nano- particles complexes explore optical, magnetic and structural properties that the single molecules do not possess. This invites the multidisciplinary approach and strategies, which includes Physics, Chemistry and Materials Science. The multifunctional nano particle complexes used for the cancer imaging purposes usually involve: (1) encapsulation and/or (2) covalent or non covalent binding of components that allows the nano- particles to recognize or locate the cancer affected area, permit imaging of the tumor and kill the tumor cells.

\author{
Mihir J Joshi \\ Department of Physics, Saurashtra University, India
}

Correspondence: Mihir J Joshi, Crystal Growth Laboratory, Department of Physics, Saurashtra University, Rajkot 360 005, India,Tel 028I 2588428,Email mshilp24@rediffmail.com

Received: December 04, 2014 | Published: December 17, 2014

The size of nano particles matters in the imaging. There is a need of nano- particles of the particular size range for EPR (Enhanced Permeation and Retention) effect. The nano- particles only of a specific range can diffuse through the endothelium of tumor tissues to experience the EPR effect. Generally the nano- particles larger than $10 \mathrm{~nm}$ are preferred to avoid the first pass elimination in the kidneys but smaller than $150 \mathrm{~nm}$ to $200 \mathrm{~nm}$ are used to avoid clearing by the liver and spleen. ${ }^{8}$

One of the major areas of nano- particles research deals with the cardiovascular diseases (CVD), which includes the targeted imaging of atherosclerosis, restenosis and CVD conditions. The targets for the detection of and imaging of atherosclerotic plaque include fibrin, tissue factor, endothelia, macrophages, collagen III and angiogenesis marker. ${ }^{9}$ The use of multifunctional nano- particles complexes, conjugated with cell specific ligand, makes it possible to deliver therapies directly to the plaques.

Nanomedicine may provide the solution for one of the foremost challenges faced by the pharmaceutical industry that is the drug delivery across the blood brain barrier (BBB). The BBB is the tightly packed layer of endothelial cells that surrounds the brain and keeps high molecular weight molecules away from entering. Only small number of drugs or small molecules with high lipid solubility or low molecular weight (less than 400-500 Daltons) can penetrate BBB. In as much as the nano- particles having small size and molecular weight, they are able to move across the BBB. The nano- carriers conjugated with ligands that attach to the brain endothelial cell receptors accumulate there and eventually internalized by cells on the vascular side of the brain through the mechanism of receptor mediated endocytosis. ${ }^{10}$

There is wide range of potential applications for nano-materials in medical devices, such as in bio-captors, ocular implants, artificial retinas, cerebral implants to treat pain, bio-ceramic materials, prosthetic devices, etc. Nano bio-ceramic materials with goodantimicrobial activity is important for dental and bone implants. ${ }^{11,12}$ Generally, metals and minerals are heavy, non-adsorable and toxic substances. When metals and minerals are converted in to very fine small size 
adsorable particles then therapeutically most effective and least or nontoxic form of medicine is obtained. According to traditional Indian medicine - Ayurveda- these formulations are known as bhasma, which is expected to incorporate the nano forms. ${ }^{13,14}$ The bulk toxic form dramatically changes the properties in bhasma form and finds application in medicine that needs detailed investigation. One can think of incorporating the reverse pharmacology approach to screen the nano-medicines and bhasma. ${ }^{15,16}$

Altogether, the nano-medicine is comparatively a recent intruder in the field of medicine. There are serious efforts made to define the term nano-medicine. According to the European Science Foundation (2004) the nano-medicine is defined as the science and technology of diagnosing, treating and preventing disease and traumatic injury, of relieving pain, and of preserving and improving human health, using molecular tools and molecular knowledge of the human body. Whereas, the NIH (2006) has defined the nano-medicine as an off shoot of nano-technology, which refers to highly scientific medical intervention at the molecular scale for curing disease or repairing damaged tissues, such as bone, muscle or nerve. ${ }^{17}$

The nano- medicine pathway is not always smooth. It requires detailed study from toxicity point of view and possible side effects, which are not yet seriously thought of. In the January 2010 an article appeared in Scientific American regarding the nano-bacteria and the authors noted that the occurrence of natural nano-particles in body has serious consequences many times. ${ }^{18}$ In the editorial of the Scientific American (January 2010) under the title, "Nano Risks: A Big Need for a Little Testing", it is warned that the elements at microscopic levels exhibiting different properties than their bulk properties, the artificially introduced nano-particles may not have beneficial effects in the human body all the times.

Today the global nano- medicine market has reached over 100 billion USD and expected to reach 177.60 billion USD in 2019. This requires multi-disciplinary approach rather than remaining confined to a particular discipline or disciplines.

\section{Conclusion}

In concluding remark, it is noted that the nano-medicine field is quite promising field with many opportunities and challenges, which needs multi-dimensional approach. This has become the field of interdisciplinary nature and needs the combined efforts of the experts from the various fields to develop the genuine final product.

\section{Acknowledgments}

None.

\section{Conflicts of interest}

None.

\section{Rerferences}

1. Junghanns JU, Muller RH. Nanocrystal technology, drug delivery and clinical applications. Int J Nanomedicine. 2008;3(3):295-309.

2. Keck CM, Muller RH. Drug nano-crystals of poorly soluble drug produced by high pressure homogenization. Eur J Pharm Biopharma. 2006;62(1):3-16.

3. Vyas PM, Joshi MJ, Pansuriya AM, et al. Synthesis And Characterization Of N-Butyl 4-(3,4-Dimethoxyphenyl)-6-Methyl-2-Thioxo-1,2,3,4 Tetrahydropyrimidine-5-Carboxylate Nanocrystalline Particles By Water/Oil Micro emulsion Method. Int J Nanosci. 2011;10(6):1237.

4. Wagner V, Dullaart A, Bock AK, et al. The emerging nanomedicine landscape. Nat Biotechnol. 2006;24(10):1211-1217.

5. Ventola CL. The nanomedicine revolution: part 2: Current and future clinical applications. 2012;PT37(10):582-591.

6. Bhaskar S, Tian F, Stoeger T, et al. Multifunctional Nanocarriers for diagnostics, drug delivery and targeted treatment across blood-brain barrier: perspectives on tracking and neuroimaging. Part Fibre Toxicol. 2010;7:3.

7. Sajja HK, East MP, Mao H, et al. Development of multifunctional nanoparticles for targeted drug delivery and non-invasive imaging of therapeutic effect. Curr Drug Discov Technol. 2009;6(1):43-51.

8. Ledet G, Mandal TK. Nanomedicine: Emerging therapeutics for the $21^{\text {st }}$ century. US Pharm. 2012;37(3):7-11.

9. Godin B, Sakamoto JH, Serda RE, et al. Emerging applications of nanomedicine for the diagnosis and treatment of cardiovascular diseases. Trends Pharmacol Sci. 2010;31(5):199-205.

10. Bawa R. Nanoparticle-based therapeutics in humans: a survey. Nanotechnology Law \& Business. 2008;5(2):135-155.

11. Galvin P, Thompson D, Ryan KB, et al. Nanoparticle based drug delivery: Case studies for cancer and cardiovascular applications. Cell Mol Life Sci. 2012;69(3):389 - 404

12. Tank KP, Chudasama KS, Thaker VS, et al. Cobalt-doped nano hydroxyapatite: synthesis, characterization, antimicrobial and hemolytic studies. J Nano part Res. 2013;15:1644.

13. Kulkarni SS. Bhasma and Nano Medicine. Int Res $J$ of Pharma. 2013;4(4):10.

14. Chaudhary A. Ayurvedic bhasma: nanomedicine of ancient Indiaits global contemporary perspective. $J$ Biomed Nanotechnol. 2011;7(1):68-69.

15. Patwardhan B, Vaidya AD, Chorghade M, et al. Reverse Pharmacology and systems approach for Drug Discovery. Current Bioactive Compounds. 2008;4:201-212.

16. Lele RD. Beyond reverse pharmacology: Mechanism-based screening of Ayurvedic drugs. J Ayurveda Integr Med. 2010;1(4):257-265.

17. Webster TJ. Nanomedicine: what's in a definition? Int J Nanomedicine. 2006;1(2):115-116.

18. Nano Risks: A Big Need for a Little Testing. (2010). 